\title{
Independent Domination in Subcubic Graphs
}

\author{
${ }^{1}$ A. Akbari, ${ }^{1}$ S. Akbari, ${ }^{1}$ A. Doosthosseini, \\ ${ }^{1}$ Z. Hadizadeh, ${ }^{2}$ Michael A. Henning, and ${ }^{1}$ A. Naraghi \\ ${ }^{1}$ Department of Mathematical Sciences \\ Sharif University of Technology \\ Tehran, Iran \\ ${ }^{2}$ Department of Mathematics and Applied Mathematics \\ University of Johannesburg \\ Johannesburg, South Africa
}

\begin{abstract}
A set $S$ of vertices in a graph $G$ is a dominating set if every vertex not in $S$ is adjacent to a vertex in $S$. If, in addition, $S$ is an independent set, then $S$ is an independent dominating set. The independent domination number $i(G)$ of $G$ is the minimum cardinality of an independent dominating set in $G$. In 2013 Goddard and Henning [Discrete Math 313 (2013), 839-854] conjectured that if $G$ is a connected cubic graph of order $n$, then $i(G) \leq \frac{3}{8} n$, except if $G$ is the complete bipartite graph $K_{3,3}$ or the 5-prism $C_{5} \square K_{2}$. Further they construct two infinite families of connected cubic graphs with independent domination three-eighths their order. They remark that perhaps it is even true that for $n>10$ these two families are only families for which equality holds. In this paper, we provide a new family of connected cubic graphs $G$ of order $n$ such that $i(G)=\frac{3}{8} n$. We also show that if $G$ is a subcubic graph of order $n$ with no isolated vertex, then $i(G) \leq \frac{1}{2} n$, and we characterize the graphs achieving equality in this bound.
\end{abstract}

Keywords: Independent domination; Cubic graph; Subcubic graph

AMS subject classification: $05 \mathrm{C} 69$

\footnotetext{
${ }^{0}$ E-mail Addresses: ami.akbari99@student.sharif.edu, s_akbari@sharif.edu, a.doosth77@student.sharif.edu, zahra.hadizadeh78@student.sharif.edu, mahenning@uj.ac.za, a. naraghi2015@student.sharif.edu
} 


\section{Introduction}

A set $S$ of vertices in a graph $G$ is a dominating set if every vertex not in $S$ is adjacent to a vertex in $S$. If, in addition, $S$ is an independent set, then $S$ is an independent dominating set, abbreviated ID-set. The independent domination number, denoted $i(G)$, of $G$ is the minimum cardinality of an ID-set in $G$. The concept of independent domination number of graphs is studied extensively in the literature, for example see [1, 2, 3, 4, 5, 7, 8, 9, 10, 11, 13, 14, 15, 16, 17, 18, 19, 20]. A survey on independent domination in graphs can be found in 6 .

For notation and graph theory terminology we generally follow [12]. The order of a graph $G$ with vertex set $V(G)$ and edge set $E(G)$ is denoted by $n(G)=|V(G)|$ and its size by $m(G)=|E(G)|$. Two vertices are neighbors in $G$ if they are adjacent. The open neighborhood of a vertex $v$ in $G$ is the set of neighbors of $v$, denoted $N_{G}(v)$. Thus, $N_{G}(v)=$ $\{u \in V(G) \mid u v \in E(G)\}$. The closed neighborhood of $v$ is the set $N_{G}[v]=N_{G}(v) \cup\{v\}$. The degree of a vertex $v$ in $G$ is denoted $d_{G}(v)=\left|N_{G}(v)\right|$. We denote the minimum and maximum degrees among the vertices of $G$ by $\delta(G)$ and $\Delta(G)$, respectively. A cubic graph is a graph in which every vertex has degree 3 , while a subcubic graph is a graph with maximum degree at most 3 .

Further, the subgraph obtained from $G$ by deleting all vertices in $S$ and all edges incident with vertices in $S$ is denoted by $G-S$. If $S=\{v\}$, we simply denote $G-\{v\}$ by $G-v$. A leaf of a graph $G$ is a vertex of degree 1 in $G$, while a support vertex of $G$ is a vertex adjacent to a leaf. A star is the graph $K_{1, k}$, where $k \geq 1$; that is, a star is a tree with at most one vertex that is not a leaf. A double star is a tree with exactly two (adjacent) non-leaf vertices. Further if one of these vertices is adjacent to $r$ leaves and the other to $s$ leaves, then we denote the double star by $S(r, s)$. We denote the path and cycle on $n$ vertices by $P_{n}$ and $C_{n}$, respectively, and we denote a complete bipartite with partite sets of cardinalities $n$ and $m$ by $K_{n, m}$. The corona $\operatorname{cor}(G)$ of a graph $G$, also denoted $G \circ P_{1}$ in the literature, is the graph obtained from $G$ by adding a pendant edge to each vertex of $G$. For $k \geq 1$ an integer, we use the standard notation $[k]=\{1, \ldots, k\}$ and $[k]_{0}=\{0,1, \ldots, k\}$.

\section{Motivation and Known Results}

As remarked in [6], since every bipartite graph is the union of two independent sets, each of which dominates the other, we have the following well-known bound on the independent domination number of a bipartite graph.

Proposition 1 If $G$ is a bipartite graph with no isolated vertices of order $n$, then $i(G) \leq \frac{1}{2} n$.

As noted in [6], the bound in Proposition 1 is sharp as may be seen by taking $G=K_{k, k}$ for any $k \geq 1$. In particular, if $G=K_{k, k}$ and $k \in[3]$, then $G$ is a connected subcubic graph of order $n=2 k$ such that $i(G)=\frac{1}{2} n$. 
It remains an open problem to determine best possible upper bounds on the independent domination number of a connected cubic graph in terms of its orders. Lam, Shiu, and Sun [14] proved that if $G$ is a connected cubic graph of order $n$ different from $K_{3,3}$, then $i(G) \leq \frac{2}{5} n$, where the graph $K_{3,3}$ is given in Figure 1(a). This bound is achieved by the 5-prism $C_{5} \square K_{2}$ which is illustrated in Figure 1(b).

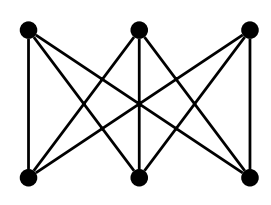

(a) $K_{3,3}$

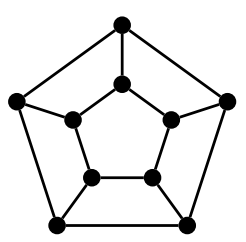

(b) $C_{5} \square K_{2}$

Figure 1: The graphs $K_{3,3}$ and $C_{5} \square K_{2}$.

Goddard and Henning [6] posed the conjecture that the $\frac{2}{5} n$ bound on the independent domination number can be improved if we forbid the exceptional graphs $K_{3,3}$ and $C_{5} \square K_{2}$.

Conjecture 1 ([6]) If $G \notin\left\{K_{3,3}, C_{5} \square K_{2}\right\}$ is a connected, cubic graph of order $n$, then $i(G) \leq \frac{3}{8} n$.

Dorbec, Henning, Montassier, and Southey [3] proved Conjecture 1 in the case when there is no subgraph isomorphic to $K_{2,3}$. In general, however, Conjecture 1 remains unresolved.

Goddard and Henning [6] constructed two infinite families $\mathcal{G}_{\text {cubic }}$ and $\mathcal{H}_{\text {cubic }}$ of connected cubic graphs with independent domination number three-eighths their orders as follows. For $k \geq 1$, a graph in the family $\mathcal{G}_{\text {cubic }}$ is constructed by taking two copies of the cycle $C_{4 k}$ with respective vertex sequences $a_{1} b_{1} c_{1} d_{1} \ldots a_{k} b_{k} c_{k} d_{k}$ and $w_{1} x_{1} y_{1} z_{1} \ldots w_{k} x_{k} y_{k} z_{k}$, and joining $a_{i}$ to $w_{i}, b_{i}$ to $x_{i}, c_{i}$ to $z_{i}$, and $d_{i}$ to $y_{i}$ for each $i \in[k]$. For $\ell \geq 1$, a graph in the family $\mathcal{H}_{\text {cubic }}$ is constructed by taking a copy of a cycle $C_{3 \ell}$ with vertex sequence $a_{1} b_{1} c_{1} \ldots a_{\ell} b_{\ell} c_{\ell}$, and for each $i \in[\ell]$, adding the vertices $\left\{w_{i}, x_{i}, y_{i}, z_{i}^{1}, z_{i}^{2}\right\}$, and joining $a_{i}$ to $w_{i}, b_{i}$ to $x_{i}$, and $c_{i}$ to $y_{i}$, and further for each $j \in[2]$, joining $z_{i}^{j}$ to each of the vertices $w_{i}, x_{i}$, and $y_{i}$. Graphs in the families $\mathcal{G}_{\text {cubic }}$ and $\mathcal{H}_{\text {cubic }}$ are illustrated in Figure 2(a) and 2(b), respectively.

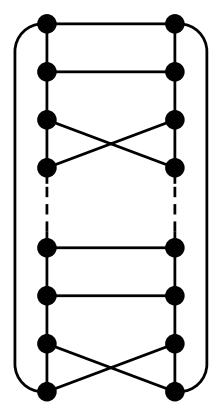

(a) $G$

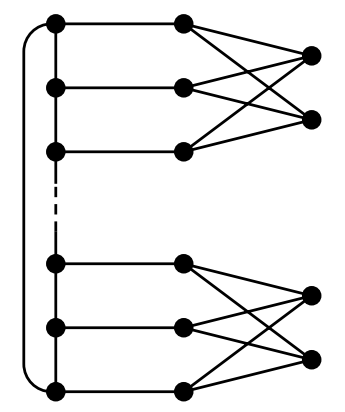

(b) $H$

Figure 2: Graphs $G \in \mathcal{G}_{\text {cubic }}$ and $H \in \mathcal{H}_{\text {cubic }}$ of order $n$ with $i(G)=i(H)=\frac{3}{8} n$. 
Theorem 1 ([6]) If $G \in \mathcal{G}_{\text {cubic }} \cup \mathcal{H}_{\text {cubic }}$ has order $n$, then $i(G)=\frac{3}{8} n$.

It is remarked in [6] that "Perhaps even more than Conjecture 1 is true, in that the only extremal graphs are those in $\mathcal{G}_{\text {cubic }} \cup \mathcal{H}_{\text {cubic }}$. We have confirmed by computer search that this is true when $n \leq 20$."

We remark that several papers, see for example [1, 2, 3, 11, 19], in which upper bounds are obtained on the independent domination number of cubic graphs present more general results on subcubic graphs.

\section{Main Results}

In this paper we have two immediate aims. Our first aim is to provide a new family of connected cubic graphs, different from the families $\mathcal{G}_{\text {cubic }}$ and $\mathcal{H}_{\text {cubic }}$, such that every graph $G$ of order $n$ in the family satisfies $i(G)=\frac{3}{8} n$. We shall prove the following result, where $\mathcal{F}_{\text {cubic }}$ is the family of connected cubic graphs constructed in Section 4 .

Theorem 2 If $G \in \mathcal{F}_{\text {cubic }}$ has order $n$, then $n \geq 16$ and $n \equiv 0(\bmod 8)$ and $i(G)=\frac{3}{8} n$.

Our second aim is to provide a tight upper bound on the independent domination number of a subcubic graph, and to characterize the graphs achieving equality in this bound. Let $G_{1} \cong K_{2,2}$ and $G_{2} \cong K_{3,3}$, and let $G_{3}, G_{4}, G_{5}$ be the three graphs shown in Figure 3 .

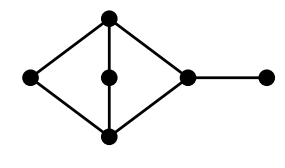

(a) $G_{3}$

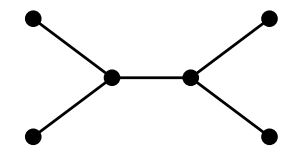

(b) $G_{4}$

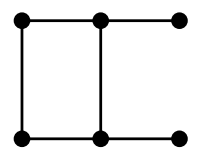

(c) $G_{5}$

Figure 3: The graphs $G_{3}, G_{4}$ and $G_{5}$

We shall prove the following result, a proof of which is presented in Section 4 .

Theorem 3 If $G$ is a subcubic graph of order $n$ with no isolated vertex, then $i(G) \leq \frac{1}{2} n$, with equality if and only if the following holds.

(a) $G \in\left\{G_{1}, G_{2}, G_{3}, G_{4}, G_{5}\right\}$.

(b) $n=2 k$ for some $k \geq 1$ and $G=\operatorname{cor}\left(P_{k}\right)$.

(c) $n=2 k$ for some $k \geq 3$ and $G=\operatorname{cor}\left(C_{k}\right)$.

\section{Proof of Theorem 2}

Let $X$ and $Y$ be the graphs shown in Figure 4(a) and 4(b), respectively. 


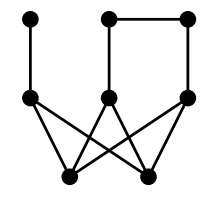

(a) $X$

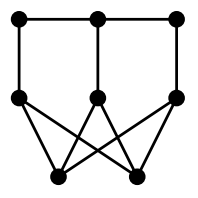

(b) $Y$

Figure 4: The graphs $X$ and $Y$

Let $\mathcal{F}_{\text {cubic }}$ be the family of graphs constructed as follows. A graph $G$ in the family $\mathcal{F}_{\text {cubic }}$ is constructed as follows. We start by taking a cycle $C: v_{1} v_{2} \ldots v_{k} v_{1}$ on $k \geq 2$ (for $k=2$

we mean two vertices adjacent with two different edges) vertices and coloring every vertex on the cycle $C$, red or blue in such a way that the number of red vertices is even. We then replace each red vertex on $C$ with a copy of $X$, and each blue vertex on $C$ with a copy of $Y$. (In the case $k=2$ we only replace each vertex by a copy of $Y$.) We call each resulting copy of $X$ and $Y$ an $X$-copy and $Y$-copy of $G$, respectively. Let $G_{i}$ be the $X$-copy or $Y$-copy associated with the vertex $v_{i}$ on the cycle $C$ for each $i \in[k]$. Thus if $v_{i}$ is colored red, then $G_{i} \cong X$, while if $v_{i}$ is colored blue, then $G_{i} \cong Y$ for $i \in[k]$. We note that there are an even number of $X$-copies in $G$. Next we partition these $X$-copies into pairs. For each resulting pair $\left\{X_{1}, X_{2}\right\}$ where $X_{i} \cong X$ for $i \in[2]$, we add two edges as follows: If $v_{i 1}$ and $v_{i 2}$ denote the two (adjacent) vertices of degree 2 in $X_{i}$ for $i \in[2]$, then we add the edges $v_{11} v_{21}$ and $v_{12} v_{22}$ as illustrated in Figure 5 .

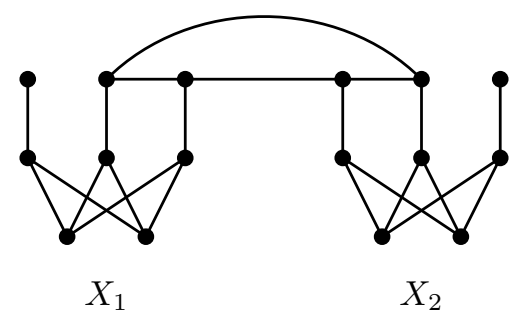

Figure 5: Joining of the pairs $X_{1}$ and $X_{2}$

We note that each $X$-copy of $G$ contains a vertex of degree 1 and each $Y$-copy of $G$ contains two vertices of degree 2. We now complete the construction of the graph $G$ as follows. Consider the subgraphs $G_{i}$ and $G_{i+1}$ where addition is taken modulo $k$ and where $i \in[k]$. If $G_{i}$ is an $X$-copy, then let $x_{i}$ denote the vertex of degree $1 \mathrm{in} G_{i}$, while if $G_{i}$ is a $Y$-copy, then let $y_{i}^{1}$ and $y_{i}^{2}$ denote the two vertices of degree 2 in $G_{i}$. If both $G_{i}$ and $G_{i+1}$ are $X$-copies, then add the edge $x_{1} x_{2}$. If both $G_{i}$ and $G_{i+1}$ are $Y$-copies, then add the edge $y_{i}^{2} y_{i+1}^{1}$. If $G_{i}$ is an $X$-copy and $G_{i+1}$ is a $Y$-copy, then add the edge $x_{1} y_{i+1}^{1}$. If $G_{i}$ is a $Y$-copy and $G_{i+1}$ is an $X$-copy, then add the edge $y_{i}^{2} x_{i+1}$. We do this for each $i \in[k]$, and let $G$ denote the resulting graph. An example of a graph $G$ in the family $\mathcal{F}_{\text {cubic }}$ constructed from a colored 7-cycle (here $k=7$ ) with four red vertices and three blue vertices is illustrated in Figure 6 .

We are now in a position to prove Theorem 2, Recall its statement.

Theorem 2. If $G \in \mathcal{F}_{\text {cubic }}$ has order $n$, then $n \geq 16$ and $n \equiv 0(\bmod 8)$ and $i(G)=\frac{3}{8} n$. 

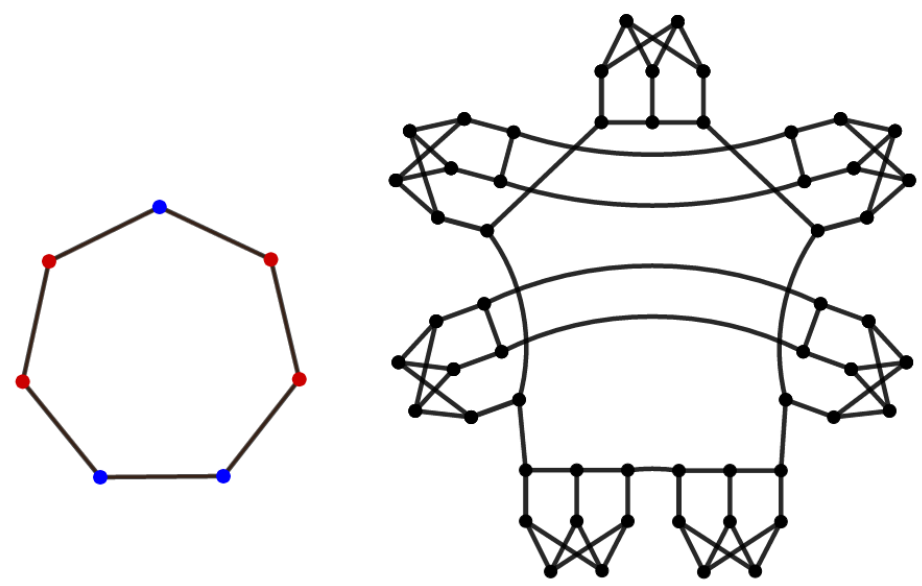

Figure 6: A graph $G$ in the family $\mathcal{F}_{\text {cubic }}$ constructed from a colored 7-cycle

Proof. If $G \in \mathcal{F}_{\text {cubic }}$ has order $n$, then by construction $G$ is obtained from a $k$-cycle for some $k \geq 2$ by replacing each vertex with a copy of $X$ or $Y$ and adding certain edges to produce a connected cubic graph. Since each copy of $X$ and $Y$ has order 8, we note that $n=8 k$. Thus, $n \geq 16$ and $n \equiv 0(\bmod 8)$. Next we show that $i(G)=\frac{3}{8} n$.

Let $S$ be an arbitrary ID-set in $G$. We show that $S$ contains at least three vertices from every $X$-copy and $Y$-copy in $G$. First we consider an $X$-copy in $G$, and let the vertices in this $X$-copy be named as in Figure 7. For notational convenience, we simply call this subgraph $X$. We show that $|S \cap V(X)| \geq 3$.

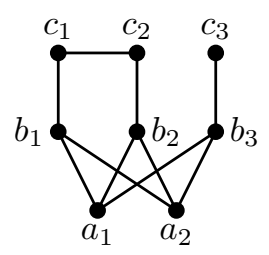

Figure 7: An $X$-copy in $G$

If $\left\{a_{1}, a_{2}\right\} \cap S=\varnothing$, then in order to dominate the vertex $b_{i}$, we note that either $b_{i} \in S$ or $c_{i} \in S$. Thus, $\left|\left\{b_{i}, c_{i}\right\} \cap S\right|=1$ for all $i \in[3]$, implying that $|S \cap V(X)| \geq 3$, as desired. Hence we may assume that $\left|\left\{a_{1}, a_{2}\right\} \cap S\right| \geq 1$, for otherwise the desired result follows. Renaming $a_{1}$ and $a_{2}$ if necessary, we may further assume that $a_{1} \in S$. Since $S$ is an independent set, we note that $\left\{b_{1}, b_{2}, b_{3}\right\} \cap S=\varnothing$, implying that $a_{2} \in S$. We show that the set $S$ contains at least one vertex from the set $\left\{c_{1}, c_{2}, c_{3}\right\}$. Suppose, to the contrary, that $\left\{c_{1}, c_{2}, c_{3}\right\} \cap S=\varnothing$. By the construction, $c_{i}$ has exactly one neighbor $c_{i}^{\prime}$ in $G \backslash V(X)$ for $i \in[2]$. In order to dominate the vertices $c_{1}$ and $c_{2}$, our earlier observations imply that $c_{1}^{\prime}, c_{2}^{\prime} \in S$. However by construction, the vertices $c_{1}^{\prime}$ and $c_{2}^{\prime}$ are adjacent, implying that the set $S$ contains two adjacent vertices, contradicting the fact that $S$ is an independent set. Hence, $\left\{c_{1}, c_{2}, c_{3}\right\} \cap S \neq \varnothing$, implying that $|S \cap V(X)| \geq 3$, as desired. 
Next we consider a $Y$-copy in $G$, and let the vertices in this $Y$-copy be named as in Figure 8. For notational convenience, we simply call this subgraph $Y$. We show that $|S \cap V(Y)| \geq 3$.

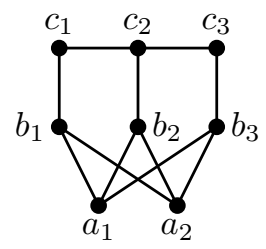

Figure 8: A $Y$-copy in $G$

If $\left\{a_{1}, a_{2}\right\} \cap S=\varnothing$, then as observed earlier, $\left|\left\{b_{i}, c_{i}\right\} \cap S\right|=1$ for all $i \in$ [3], implying that $|S \cap V(X)| \geq 3$, as desired. Hence we may assume that $\left|\left\{a_{1}, a_{2}\right\} \cap S\right| \geq 1$, for otherwise the desired result follows. Further we may assume that $a_{1} \in S$. As observed earlier, this implies that $\left\{b_{1}, b_{2}, b_{3}\right\} \cap S=\varnothing$ and $a_{2} \in S$. In order to dominate the vertex $c_{2}$, the set $S$ contains at least one of the vertices $c_{1}, c_{2}$ and $c_{3}$. Thus, $\left|\left\{c_{1}, c_{2}, c_{3}\right\} \cap S\right| \geq 1$, and so $|S \cap V(Y)| \geq 3$, as desired. This completes the proof of Theorem 2 .

\section{$5 \quad$ Proof of Theorem 3}

In this section, we present a proof of Theorem 3 . First we prove that the independent domination number of a subcubic graph with no isolated vertex is at most one-half the order of the graph.

Theorem 4 If $G$ is a subcubic graph of order $n$ with no isolated vertex, then $i(G) \leq \frac{1}{2} n$.

Proof. By linearity, the independent domination number of a graph is the sum of the independent domination numbers of its components. Hence it suffices for us to prove the bound for connected graphs; that is, we prove that if $G$ is a connected subcubic graph of order $n \geq 2$, then $i(G) \leq \frac{1}{2} n$. We proceed by induction on the order $n \geq 2$. If $n=2$, then $G=K_{2}$ and $i(G)=1=\frac{1}{2} \times 2$. If $n=3$, then $G=K_{3}$ or $G=P_{3}$, and in both cases, $i(G)=1<\frac{1}{2} \times 3$. This establishes the base cases. Let $n \geq 4$ and assume that if $G^{\prime}$ is a connected subcubic graph of order $n^{\prime}$ where $2 \leq n^{\prime}<n$, then $i\left(G^{\prime}\right) \leq \frac{1}{2} n^{\prime}$. Let $G$ be a connected subcubic graph of order $n$. If $G$ is a bipartite graph, then the desired bound follows from Proposition 1. Hence we may assume that $G$ contains an odd cycle $C$.

First, assume that there exists a vertex $u$ on the cycle $C$ with a leaf neighbor, say $w$, and consider the graph $H=G-\{u, w\}$. Since the two neighbors of $u$ on the cycle $C$ are connected in $H$ by the path $C-u$, the graph $H$ is a connected subcubic graph. Since $n \geq 4$, we note that $|V(H)|=n-2 \geq 2$. Applying the induction to $H$, we have $i(H) \leq \frac{1}{2}|V(H)|=\frac{1}{2}(n-2)$. Every minimum ID-set in $H$ can be extended to an ID-set of $G$ by adding to it the vertex $w$, implying that $i(G) \leq i(H)+1 \leq \frac{1}{2} n$. Hence we may assume that no vertex on the cycle $C$ has a leaf neighbor. 
Next, assume there are two consecutive vertices, say $u$ and $w$, on the cycle $C$ both of degree 2 in $G$. We now consider the graph $H=G-\{u, w\}$. We note that the graph $H$ is a connected subcubic graph. Further since $n \geq 4$, we note that $|V(H)|=n-2 \geq 2$. Applying the induction to $H$, we have $i(H) \leq \frac{1}{2}|V(H)|=\frac{1}{2}(n-2)$. Let $S^{\prime}$ be a minimum ID-set of $H$, and let $u^{\prime}$ be the neighbor of $u$ different from $w$ and let $w^{\prime}$ be the neighbor of $w$ different from $u$. (Possibly, $u^{\prime}=w^{\prime}$.) If $u^{\prime} \notin S^{\prime}$, then let $S=S^{\prime} \cup\{u\}$. If $u^{\prime} \in S$ and $w^{\prime} \notin S$, then let $S=S^{\prime} \cup\{w\}$. If $u^{\prime} \in S$ and $w^{\prime} \in S$, then let $S=S^{\prime}$. In all cases, $S$ is an ID-set of $G$, and so $i(G) \leq|S| \leq\left|S^{\prime}\right|+1=i(H)+1 \leq \frac{1}{2} n$. Hence we may assume that no two consecutive vertices on the cycle $C$ both have degree 2 in $G$.

Let $u$ and $v$ be two arbitrary consecutive (adjacent) vertices on the cycle $C$. Suppose that there exists a vertex $w$ of degree 2 adjacent to both $u$ and $v$. In this case, we consider the connected subcubic graph $H=G-\{u, v, w\}$. If $|V(H)|=1$, then $n=4$ and $G \cong K_{4}-e$ where $e$ is the missing edge of the complete graph $K_{4}$. In this case, $i(G)=1<\frac{1}{2} \times 4$. Hence we may assume that $|V(H)| \geq 2$. Applying the induction to $H$ we have $i(H) \leq \frac{1}{2}|V(H)|=$ $\frac{1}{2}(n-3)$. Every minimum ID-set of $H$ can be extended to an ID-set of $G$ by adding to it the vertex $w$, implying that $i(G) \leq i(H)+1<\frac{1}{2} n$. Hence we may assume that there is no vertex of degree 2 adjacent to both $u$ and $v$.

We now consider the subcubic graph $H=G-\{u, v\}$. With our assumptions, we note that $H$ has at most three components, each of which has order at least 2 . Let $H_{1}, \ldots, H_{t}$ be the components of $H$, and so $t \leq 3$. Let $S_{i}$ be a minimum ID-set of $H_{i}$ for $i \in[t]$. By the inductive hypothesis, $\left|S_{i}\right| \leq \frac{1}{2}\left|V\left(H_{i}\right)\right|$ for $i \in[t]$. Let

$$
S^{\prime}=\bigcup_{i=1}^{t} S_{i} .
$$

If $u$ has no neighbor in $S^{\prime}$, then let $S=S^{\prime} \cup\{u\}$. If $u$ has a neighbor in $S^{\prime}$ and $v$ has no neighbor in $S^{\prime}$, then let $S=S^{\prime} \cup\{v\}$. If both $u$ and $v$ have a neighbor in $S^{\prime}$, then let $S=S^{\prime}$. In all three cases, the set $S$ is an ID-set of $G$, and so $i(G) \leq|S| \leq\left|S^{\prime}\right|+1 \leq \frac{1}{2}|V(H)|+1=\frac{1}{2} n$. This completes the proof of Theorem 4 .

We are now in a position to present a proof of Theorem 3. Recall its statement.

Theorem 3. If $G$ is a subcubic graph of order $n$ with no isolated vertex, then $i(G) \leq \frac{1}{2} n$. Further, if $G$ is connected, then equality holds if and only if the following holds.

(a) $G \in\left\{G_{1}, G_{2}, G_{3}, G_{4}, G_{5}\right\}$.

(b) $n=2 k$ for some $k \geq 1$ and $G=\operatorname{cor}\left(P_{k}\right)$.

(c) $n=2 k$ for some $k \geq 3$ and $G=\operatorname{cor}\left(C_{k}\right)$.

Proof. The upper bound $i(G) \leq \frac{1}{2} n$ is a restatement of Theorem 4 . If $G$ is a connected subcubic graph of order $n$ that satisfies (a), (b) or (c) in the statement of the theorem, then it is a simple exercise to check that $i(G)=\frac{1}{2} n$. Hence it suffices to prove that if $G$ is a connected subcubic graph of order $n \geq 2$ satisfying $i(G)=\frac{1}{2} n$, then (a), (b) or (c) in the statement of the theorem hold. We proceed by induction on the order $n \geq 2$. We note that $n=2 i(G)$ is even since $i(G)$ is an integer. If $n=2$, then $G=K_{2}=\operatorname{cor}\left(P_{1}\right)$. Suppose that 
$n=4$. If $\Delta(G)=3$, then $i(G)=1<\frac{1}{2} \times 4$, a contradiction. Hence, $\Delta(G)=2$, and so either $G=P_{4}=\operatorname{cor}\left(P_{2}\right)$ or $G=K_{2,2}=G_{1}$. This establishes the base cases. Let $n \geq 6$ be even and assume that if $G^{\prime}$ is a connected subcubic graph of even order $n^{\prime}$ where $2 \leq n^{\prime}<n$ satisfying $i\left(G^{\prime}\right)=\frac{1}{2} n^{\prime}$, then (a), (b) or (c) in the statement of the theorem hold. Let $G$ be a connected subcubic graph of order $n$ satisfying $i(G)=\frac{1}{2} n$. We proceed further with two claims. Recall that $G_{2}=K_{3,3}$.

Claim 1 If the graph $G$ contains no support vertex, then $G=G_{2}$.

Proof. Assume that the graph $G$ contains no support vertex. Thus, every vertex of $G$ has degree at least 2 and degree at most 3 . If $\Delta(G)=2$, then $G$ is a cycle $C_{n}$ where $n \geq 6$, and so $i(G)=i\left(C_{n}\right)=\left\lceil\frac{1}{3} n\right\rceil<\frac{1}{2} n$, a contradiction. Hence, $\Delta(G)=3$. Let $v$ be an arbitrary vertex of degree 3 in $G$ and let $N_{G}(v)=\{x, y, z\}$. We now consider the graph $H=G \backslash N_{G}[v]$. Suppose that $H$ has no isolated vertex. Let $H_{1}, \ldots, H_{t}$ be the components of $H$ and let $S_{i}$ be a minimum ID-set of $H_{i}$ for $i \in[t]$. By Theorem $4,\left|S_{i}\right|=i\left(H_{i}\right) \leq \frac{1}{2}\left|V\left(H_{i}\right)\right|$ for $i \in[t]$. Let

$$
S^{\prime}=\bigcup_{i=1}^{t} S_{i} \quad \text { and } \quad S=S^{\prime} \cup\{v\} .
$$

The set $S$ is an ID-set of $G$, implying that

$$
\begin{aligned}
i(G) \leq|S|=1+\left|S^{\prime}\right| & =1+\sum_{i=1}^{t}\left|S_{i}\right| \\
& \leq 1+\sum_{i=1}^{t} \frac{1}{2}\left|V\left(H_{i}\right)\right| \\
& =1+\frac{1}{2}|V(H)| \\
& =1+\frac{1}{2}(n-4) \\
& <\frac{1}{2} n,
\end{aligned}
$$

a contradiction. Hence, $H$ contains at least one isolated vertex. If $H$ contains at least four isolated vertices, then since $\delta(G) \geq 2$ each such isolated vertex in $H$ has at least two neighbors in $G$ belonging to the set $\{x, y, z\}$, implying by the Pigeonhole Principle that at least one of the vertices $x, y$ and $z$ has degree at least 4 in $G$, a contradiction. Therefore, $H$ contains at most three isolated vertices.

We show that $H$ contains at most two isolated vertices. Suppose, to the contrary, that $H$ contains three isolated vertices. Since $\delta(G) \geq 2$ and $\Delta(G)=3$, the graph $G$ is now determined. In this case, $n=7$ and this contradicts the fact that $n$ is even. Hence, $H$ contains at most two isolated vertices.

Next we show that $H$ contains exactly two isolated vertices. Suppose, to the contrary, that $H$ contains exactly one isolated vertex, say $u$. In this case, we consider the graph 
$H^{\prime}=H \backslash\{u\}$. Since $n \geq 6$ is even and $H^{\prime}$ contains no isolated vertex, every component of $H^{\prime}$ has order at least 2. Applying Theorem 4 to $H^{\prime}$, we have $i\left(H^{\prime}\right) \leq \frac{1}{2}\left|V\left(H^{\prime}\right)\right|=\frac{1}{2}(n-5)$. Since $n$ is even, this implies that $i\left(H^{\prime}\right) \leq \frac{1}{2}(n-6)$. A minimum ID-set of $H^{\prime}$ can be extended to an ID-set of $G$ by adding to it the vertices $u$ and $v$, implying that $i(G) \leq i\left(H^{\prime}\right)+2<\frac{1}{2} n$, a contradiction. Hence, $H$ contains exactly two isolated vertices.

Let $u$ and $w$ be the two isolated vertices of $H$. Each of $u$ and $w$ has either two or three neighbors in $G$ that belong to the set $\{x, y, z\}$, implying that $u$ and $w$ have at least one common neighbor.

Suppose that $u$ and $w$ have exactly one common neighbor. Renaming the neighbors of $v$ if necessary, we may assume that $N_{G}(u)=\{x, y\}$ and $N_{G}(w)=\{y, z\}$. In particular, $y$ is the common neighbor of $u$ and $w$. Let $H^{\prime}=H-\{u, w\}$. We note that $H^{\prime}$ has no isolated vertex. Applying Theorem 4, $i\left(H^{\prime}\right) \leq \frac{1}{2}\left|V\left(H^{\prime}\right)\right|=\frac{1}{2}(n-6)$. Let $S$ be a minimum ID-set of $H^{\prime}$. If $N(z) \cap S=\varnothing$, then $S \cup\{u, z\}$ is an ID-set of G, a contradiction. If $N(x) \cap S=\varnothing$, then similarly we get a contradiction. Now, assume that $N(z) \cap S \neq \varnothing$ and $N(x) \cap S \neq \varnothing$. In this case, $S \cup y$ is an ID-set of $G$, a contradiction. Hence, $u$ and $w$ have at least two common neighbors.

Suppose that $u$ and $w$ have exactly two common neighbors. Renaming neighbors of $v$ if necessary, we may assume in this case that $\{x, y\}=N_{G}(u) \cap N_{G}(w)$. By assumption, $z$ is adjacent to at most one of $u$ and $w$. Renaming $u$ and $w$, we may assume that $z$ is not adjacent to $w$. If $n=6$, then $\{w, z\}$ is an ID-set of $G$, implying that $i(G)=2<\frac{1}{2} \times 6$, a contradiction. Hence, $n \geq 8$. We now consider the connected subcubic graph $H^{\prime}=G-\{u, v, w, x, y\}$. Applying Theorem 4 to $H^{\prime}$, we have $i\left(H^{\prime}\right) \leq \frac{1}{2}\left|V\left(H^{\prime}\right)\right|=\frac{1}{2}(n-5)$. Since $n$ is even, this implies that $i\left(H^{\prime}\right) \leq \frac{1}{2}(n-6)$. A minimum ID-set of $H^{\prime}$ can be extended to an ID-set of $G$ by adding to it the vertices $x$ and $y$, implying that $i(G) \leq i\left(H^{\prime}\right)+2<\frac{1}{2} n$, a contradiction.

Hence, the vertices $u$ and $w$ have three common neighbors. The graph $G$ is now determined, and $G=K_{3,3}=G_{2}$. This completes the proof of the claim. (ㅁ)

By Claim 1, we may assume that the graph $G$ contains at least one support vertex, for otherwise $G=G_{2}$ and the desired result follows. Since $n \geq 6$, we note that every support vertex of $G$ has at most two leaf neighbors. Recall that $G_{4}$ is the double star $S(2,2)$ shown in Figure 3(b).

Claim 2 If the graph $G$ contains a support vertex with two leaf neighbors, then $G=G_{4}$.

Proof. Suppose that $G$ contains a support vertex $v$ with two leaf neighbors, say $u$ and $w$. Let $x$ be the third neighbor of $v$. Since $n \geq 6$, we note that $d_{G}(x) \geq 2$. We show that $x$ is a support vertex. Suppose, to the contrary, that $x$ is not a support vertex. In this case, we consider the subcubic graph $H=G-N_{G}[v]=G-\{u, v, w, x\}$. Since $x$ is not a support vertex in $G$, every component of $H$ has order at least 2. Applying Theorem 4 to $H$, we have $i(H) \leq \frac{1}{2}|V(H)|=\frac{1}{2}(n-4)$. A minimum ID-set of $H$ can be extended to an ID-set of $G$ by adding to it the vertex $v$, implying that $i(G) \leq i(H)+1<\frac{1}{2} n$, a contradiction. Hence, $x$ is a support vertex. 
Next, we show that $x$ has two leaf neighbors. Suppose, to the contrary, that $x$ has exactly one leaf neighbor, say $y$. Since $n \geq 6$, we note that in this case the vertex $x$ has degree 3 . We consider the connected subcubic graph $H=G-\{u, v, w, x, y\}$. We note that $H$ has order at least 2. Applying Theorem 4 to $H$, we have $i(H) \leq \frac{1}{2}|V(H)|=\frac{1}{2}(n-5)$. Since $n$ is even, this implies that $i(H) \leq \frac{1}{2}(n-6)$. A minimum ID-set of $H$ can be extended to an ID-set of $G$ by adding to it the vertices $v$ and $y$, implying that $i(G) \leq i(H)+2<\frac{1}{2} n$, a contradiction. Hence, $x$ has exactly two leaf neighbors; that is, $G=G_{4}$. (口)

By Claim 2, we may assume that every support of $G$ has exactly one leaf neighbor, for otherwise $G=G_{4}$ and the desired result follows. Among all support vertices of $G$, let $v$ be chosen so that the following holds, where $u$ is the leaf neighbor of $v$.

(1) The degree, $d_{G}(v)$, of $v$ is a minimum.

(2) Subject to (1), the number of components of $G-\{u, v\}$ is a minimum.

We note that either $d_{G}(v)=2$ or $d_{G}(v)=3$. Further, we note that either $G-\{u, v\}$ is connected or has two components. Let $H=G-\{u, v\}$. Each component of $H$ contains a neighbor of $v$. Since $u$ is the only leaf neighbor of $v$, the graph $H$ has no isolated vertex, and so each component of $H$ has order at least 2. Applying Theorem 4 to $H$, we have $i(H) \leq \frac{1}{2}|V(H)|=\frac{1}{2}(n-2)$. A minimum ID-set of $H$ can be extended to an ID-set of $G$ by adding to it the vertex $u$, implying that $\frac{1}{2} n=i(G) \leq i(H)+1 \leq \frac{1}{2} n$. Hence we must have equality throughout this inequality chain, implying that $i(H)=\frac{1}{2}|V(H)|$ and that every component $H^{\prime}$ of $H$ satisfies $i\left(H^{\prime}\right)=\frac{1}{2}\left|V\left(H^{\prime}\right)\right|$. Applying the inductive hypothesis to each component $H^{\prime}$ of $H$, the component $H^{\prime}$ satisfies (a), (b) or (c) in the statement of the theorem. Recall that $G_{3}$ and $G_{5}$ are the graphs shown in Figure 3(a) and 3(b), respectively.

Claim 3 The graph $H$ is connected.

Proof. Suppose, to the contrary, that $H$ is disconnected. Thus, $H$ has two components, say $H_{1}$ and $H_{2}$. In particular, this implies that $d_{G}(v)=3$. Let $v_{i}$ be the neighbor of $v$ that belongs to $H_{i}$ for $i \in[2]$. Let $H_{i}$ have order $n_{i}$ for $i \in[2]$. As observed earlier, $n_{i} \geq 2$ and $i\left(H_{i}\right)=\frac{1}{2} n_{i}$ for $i \in[2]$. Further, $H_{i}$ satisfies (a), (b) or (c) in the statement of the theorem for $i \in[2]$.

Claim 3.1 $H_{i} \notin\left\{G_{1}, G_{2}, G_{3}, G_{4}, G_{5}\right\}$ for $i \in[2]$.

Proof. Suppose, to the contrary, that $H_{1} \in\left\{G_{1}, G_{2}, G_{3}, G_{4}, G_{5}\right\}$. We note that $H_{1} \neq G_{2}=$ $K_{3,3}$ since the vertex $v_{1}$ has degree at most 2 in $H_{1}$. Thus, $H_{1}=G_{1}$, in which case $n_{1}=4$, or $H_{1} \in\left\{G_{3}, G_{4}, G_{5}\right\}$, in which case $n_{1}=6$. Further we note that $H_{1}-v_{1}$ is a connected graph of odd order $n_{1}-1 \geq 3$, implying by Theorem 4 that $i\left(H_{1}-v_{1}\right) \leq \frac{1}{2}\left(n_{1}-2\right)$. Let $S_{1}$ be a minimum ID-set of $H_{1}$. We note that the set $S_{1}$ contains no neighbor of $v_{1}$. We now consider the connected subcubic graph $G^{\prime}=G-\left(V\left(H_{1}\right) \backslash\left\{v_{1}\right\}\right)$. Let $G^{\prime}$ has order $n^{\prime}$. Since $n^{\prime}=n-n_{1}+1$ is odd, Theorem 4 implies that $i\left(G^{\prime}\right) \leq \frac{1}{2}\left(n^{\prime}-1\right)=\frac{1}{2}\left(n-n_{1}\right)$. If $S^{\prime}$ is an ID-set of $G^{\prime}$ of minimum cardinality, then $S^{\prime} \cup S_{1}$ is an ID-set of $G$, implying that

$$
i(G) \leq\left|S_{1}\right|+\left|S^{\prime}\right| \leq \frac{1}{2}\left(n_{1}-2\right)+\frac{1}{2}\left(n-n_{1}\right)<\frac{1}{2} n,
$$


a contradiction. (口)

By Claim 3.1 and our earlier observations, $H_{i}$ satisfies (b) or (c) in the statement of the theorem for $i \in[2]$. Thus, $n_{i}=2 k_{i}$ and $H_{i}=\operatorname{cor}\left(P_{k_{i}}\right)$ for some $k_{i} \geq 1$ or $H_{i}=\operatorname{cor}\left(C_{k_{i}}\right)$ for some $k_{i} \geq 3$ and $i \in[2]$. Recall that among all support vertices of $G$, the vertex $v$ was chosen to have minimum degree. This implies that if $H_{i}=\operatorname{cor}\left(P_{k_{i}}\right)$, then $k_{i} \geq 2$ for $i \in[2]$, for otherwise if $H_{i}=\operatorname{cor}\left(P_{1}\right)=P_{2}$, then the vertex $v_{i}$ would be a support vertex of $G$ of degree 2 , a contradiction. In particular, we note that $n_{i} \geq 4$ for $i \in$ [2]. If $H_{1}=\operatorname{cor}\left(P_{k_{1}}\right)$ for some $k_{1} \geq 2$, then at least one of the two support vertices of degree 2 in $H$ is a support vertex of degree 2 in $G$, contradicting our choice of the support vertex $v$. If $H_{1}=\operatorname{cor}\left(C_{k_{1}}\right)$ for some $k_{1} \geq 3$, then at least one support vertex (of degree 3 ) in $H$ is a support vertex in $G$. However, the removal of such a support vertex and its leaf neighbor in $G$ produces a connected graph, once again contradicting our choice of the support vertex $v$. This completes the proof of Claim 3 . (ㅁ)

By Claim 3, the graph $H$ is connected. Let $H$ have order $n^{\prime}$, and so $n^{\prime}=n-2$. As observed earlier, $H$ satisfies (a), (b) or (c) in the statement of the theorem. Thus, $H \in$ $\left\{G_{1}, G_{2}, G_{3}, G_{4}, G_{5}\right\}$ or $n^{\prime}=2 k^{\prime}$ for some $k^{\prime} \geq 1$ and $H=\operatorname{cor}\left(P_{k^{\prime}}\right)$ or $n^{\prime}=2 k^{\prime}$ for some $k^{\prime} \geq 3$ and $H=\operatorname{cor}\left(C_{k^{\prime}}\right)$.

Claim 4 If $H \in\left\{G_{1}, G_{2}, G_{3}, G_{4}, G_{5}\right\}$, then $G=G_{3}$.

Proof. Suppose that $H \in\left\{G_{1}, G_{2}, G_{3}, G_{4}, G_{5}\right\}$. We consider each possibility in turn. Suppose that $H=G_{1}=C_{4}$, and so $n=6$. Let $H$ be the cycle $C: w_{1} w_{2} w_{3} w_{4} w_{1}$, where $v w_{1}$ is an edge of $G$. If $v w_{3}$ is not an edge of $G$, then $\left\{v, w_{3}\right\}$ is an ID-set of $G$, and so $i(G)=2<\frac{1}{2} \times 6$, a contradiction. Hence, $v w_{3}$ is an edge of $G$, implying that $G=G_{3}$.

We note that the vertex $v$ has one or two neighbors in $H$, and each neighbor of $v$ in $H$ has degree at most 2 in $H$, implying that $H \neq G_{2} \cong K_{3,3}$.

Suppose that $H=G_{3}$, and so $n=8$. Let $a_{1}$ and $a_{2}$ be the two vertices of $H$ with three common neighbors, say $b_{1}, b_{2}$ and $b_{3}$, where $b_{3}$ has degree 3 in $H$. Let $w$ be the leaf neighbor of $b_{3}$ in $H$. If $v w \in E(G)$, then let $S=\left\{a_{1}, a_{2}, v\right\}$. If $v w \notin E(G)$ and $v$ is adjacent to both $b_{1}$ and $b_{2}$, then let $S=\left\{b_{3}, v\right\}$. If $v w \notin E(G)$ and $v$ is adjacent to exactly one of $b_{1}$ and $b_{2}$, say to $b_{1}$, then let $S=\left\{b_{2}, b_{3}, v\right\}$. In all three cases, the set $S$ is an ID-set of $G$ and $|S| \leq 3$. Thus, $i(G) \leq 3<\frac{1}{2} \times 8$, a contradiction.

Suppose that $H=G_{4}$, and so $n=8$. Let $x$ and $y$ be the two central vertices of the double star $H$. By our earlier assumptions, every support vertex of $G$ has exactly one leaf neighbor. Hence, the vertex $v$ is adjacent in $G$ to a leaf neighbor in $H$ of $x$ and a leaf neighbor in $H$ of $y$. Thus if $x^{\prime}$ be the leaf neighbor of $x$ in $H$ that is not adjacent to $v$ in $G$, then the set $\left\{v, x^{\prime}, y\right\}$ is an ID-set of $G$, and so $i(G) \leq 3<\frac{1}{2} \times 8$, a contradiction.

Suppose that $H=G_{5}$, and so $n=8$. Thus, $H$ is obtained from a path $a_{1} a_{2} a_{3} a_{4} a_{5} a_{6}$ by adding the edge $a_{2} a_{5}$. Suppose that $v$ is adjacent to $a_{1}$ or $a_{6}$, say to $a_{1}$. If $v$ is not adjacent to $a_{3}$, then let $S=\left\{v, a_{3}, a_{5}\right\}$. If $v$ is adjacent to $a_{3}$, then let $S=\left\{v, a_{5}\right\}$. In both cases, 
the set $S$ is an ID-set of $G$ and $|S| \leq 3$. Thus, $i(w 1 G) \leq 3<\frac{1}{2} \times 8$, a contradiction. Hence, $v$ is adjacent to neither $a_{1}$ nor $a_{6}$. Thus, the only possible neighbors of $v$ in $H$ are $a_{3}$ or $a_{4}$. By symmetry, we may assume that $v a_{3} \in E(G)$. Thus, $\left\{v, a_{1}, a_{5}\right\}$ is an ID-set of $G$, and so $i(G) \leq 3<\frac{1}{2} \times 8$, a contradiction. This completes the proof of Claim 4 . (ㅁ)

Let $n^{\prime}=|V(H)|$, and so $n^{\prime}=n-2$. Recall that $n \geq 6$, and so $n^{\prime} \geq 4$. By Claim 4, we may assume that $H \notin\left\{G_{1}, G_{2}, G_{3}, G_{4}, G_{5}\right\}$, for otherwise $G=G_{3}$, and the desired result follows. Hence $n^{\prime}=2 k^{\prime}$ and $H=\operatorname{cor}\left(P_{k^{\prime}}\right)$ for some $k^{\prime} \geq 2$ or $H=\operatorname{cor}\left(C_{k^{\prime}}\right)$ for some $k^{\prime} \geq 3$.

Claim $5 H=\operatorname{cor}\left(P_{k^{\prime}}\right)$ for some $k^{\prime} \geq 2$.

Proof. Suppose that $H=\operatorname{cor}\left(C_{k^{\prime}}\right)$ for some $k^{\prime} \geq 3$. Thus, $n^{\prime}=2 k^{\prime}$ and $n=2 k^{\prime}+2$. Let $H$ be the corona of the cycle $C: v_{1} v_{2} \ldots v_{k^{\prime}} v_{1}$, and let $u_{i}$ be the resulting leaf neighbor of $v_{i}$ in $H$ for $i \in\left[k^{\prime}\right]$. Since $G$ is a subcubic graph, we note that the only possible neighbors of $v$ that belong to $H$ are the leaves of $H$. We now consider the connected subcubic graph $G^{\prime}=G-N_{G}[v]$ of order at least 2. Applying Theorem 4 to the graph $G^{\prime}$, we have $i\left(G^{\prime}\right) \leq \frac{1}{2}\left|V\left(G^{\prime}\right)\right| \leq \frac{1}{2}(n-3)$. Since $n$ is even, this implies that $i\left(G^{\prime}\right) \leq \frac{1}{2}(n-4)$. A minimum ID-set of $G^{\prime}$ can be extended to an ID-set of $G$ by adding to it the vertex $v$, implying that $i(G) \leq i\left(G^{\prime}\right)+1<\frac{1}{2} n$, a contradiction. (ㅁ)

By Claim 5, $H=\operatorname{cor}\left(P_{k^{\prime}}\right)$ for some $k^{\prime} \geq 2$. Thus, $n^{\prime}=2 k^{\prime}$ and $n=2 k^{\prime}+2$. Let $H$ be the corona of the path $P: v_{1} v_{2} \ldots v_{k^{\prime}}$, and let $u_{i}$ be the resulting leaf neighbor of $v_{i}$ in $H$ for $i \in\left[k^{\prime}\right]$. Since $G$ is a subcubic graph, we note that the only possible neighbors of $v$ that belong to $H$ are the vertices $u_{i}$ for $i \in\left[k^{\prime}\right]$ or the vertices $v_{1}$ and $v_{k^{\prime}}$ of degree 2 in $H$.

Claim 6 If $d_{G}(v)=2$, then $G=\operatorname{cor}\left(P_{k}\right)$ where $k=k^{\prime}+1$.

Proof. Suppose that $d_{G}(v)=2$. Let $w$ be the neighbor of $v$ different from $u$. If $w=u_{i}$ for some $i \in\left[k^{\prime}\right]$, then we consider the connected subcubic graph $G^{\prime}=G-\{u, v, w\}$ of order at least 3. Applying Theorem 4 to the graph $G^{\prime}$, we have $i\left(G^{\prime}\right) \leq \frac{1}{2}\left|V\left(G^{\prime}\right)\right|=\frac{1}{2}(n-3)$. Since $n$ is even, this implies that $i\left(G^{\prime}\right) \leq \frac{1}{2}(n-4)$. A minimum ID-set of $G^{\prime}$ can be extended to an ID-set of $G$ by adding to it the vertex $v$, implying that $i(G) \leq i\left(G^{\prime}\right)+1<\frac{1}{2} n$, a contradiction. Hence, either $w=v_{1}$ or $w=v_{k^{\prime}}$. In both cases, $G=\operatorname{cor}\left(P_{k}\right)$ where $k=k^{\prime}+1$, as desired. (口)

By Claim 6, we may assume that $d_{G}(v)=3$. Hence, the vertex $v$ has two neighbors in $H$, say $w$ and $x$. If both neighbors $w$ and $x$ are leaves in $H$, then we consider the connected subcubic graph $G^{\prime}=G-\{u, v, w, x\}$ of order at least 2. Applying Theorem 4 to the graph $G^{\prime}$, we have $i\left(G^{\prime}\right) \leq \frac{1}{2}\left|V\left(G^{\prime}\right)\right|=\frac{1}{2}(n-4)$. A minimum ID-set of $G^{\prime}$ can be extended to an ID-set of $G$ by adding to it the vertex $v$, implying that $i(G) \leq i\left(G^{\prime}\right)+1<\frac{1}{2} n$, a contradiction. Hence, renaming $w$ and $x$ if necessary, we may assume that $w=v_{1}$.

If $x=v_{k^{\prime}}$, then $G=\operatorname{cor}\left(C_{k}\right)$ where $k=k^{\prime}+1$, and the desired result follows. Hence, we may assume that $x$ is a leaf of $H$. 
If $x=u_{1}$, then again we consider the connected subcubic graph $G^{\prime}=G-\{u, v, w, x\}$, and as before obtain the contradiction $i(G) \leq i\left(G^{\prime}\right)+|\{v\}|<\frac{1}{2} n$. Hence, $x=u_{i}$ for some $i \in\left[k^{\prime}\right] \backslash\{1\}$. Suppose that $k^{\prime} \geq 3$. In this case, we consider the connected subcubic graph $G^{\prime}=G-\left\{u, v, w, x, u_{1}\right\}$ of order at least 3. Applying Theorem 4 to the graph $G^{\prime}$, we have $i\left(G^{\prime}\right) \leq \frac{1}{2}\left|V\left(G^{\prime}\right)\right|=\frac{1}{2}(n-5)$. Since $n$ is even, this implies that $i\left(G^{\prime}\right) \leq \frac{1}{2}(n-6)$. A minimum ID-set of $G^{\prime}$ can be extended to an ID-set of $G$ by adding to it the vertices $u_{1}$ and $v$, implying that $i(G) \leq i\left(G^{\prime}\right)+2<\frac{1}{2} n$, a contradiction. Hence, $k^{\prime}=2$, implying that $G=G_{5}$, and the desired result follows. This completes the proof of Theorem 3 (ㅁ)

\section{References}

[1] G. Abrishami, and M.A. Henning, Independent domination in subcubic graphs of girth at least six, Discrete Math. 341 (2018), 155-164.

[2] C. Brause and M.A. Henning, Independent domination in bipartite cubic graphs, Graphs Combin 35(4) (2019), 881-919.

[3] P. Dorbec, M.A. Henning, M. Montassier, and J. Southey, Independent domination in cubic graphs, J. Graph Theory 80(4) (2015), 329-349.

[4] O. Favaron, A bound on the independent domination number of a tree, Vishwa Internat. J. Graph Theory 1 (1992), 19-27.

[5] M. Furuya, K. Ozeki, and A. Sasaki, On the ratio of the domination number and the independent domination number in graphs, Discrete Appl. Math. 178 (2014), 157-159.

[6] W. Goddard and M.A. Henning, Independent domination in graphs: A survey and recent results, Discrete Math. 313 (2013), 839-854.

[7] W. Goddard, M.A. Henning, J. Lyle, and J. Southey, On the independent domination number of regular graphs, Annals Combin. 16 (2012), 719-732.

[8] W. Goddard and J. Lyle, Independent dominating sets in triangle-free graphs, J. Comb. Optim. 23(1) (2012), 9-20.

[9] J. Haviland, Independent domination in regular graphs, Discrete Math. 143 (1995), $275-280$.

[10] J. Haviland, Upper bounds for independent domination in regular graphs, Discrete Math. 307 (2007), 2643-2646.

[11] M.A. Henning, C. Löwenstein, and D. Rautenbach, Independent domination in subcubic bipartite graphs of girth at least six, Discrete Appl. Math. 162 (2014), 399-403.

[12] M.A. Henning and A. Yeo, Total Domination in Graphs (Springer Monographs in Mathematics) 2013, ISBN: 978-1-4614-6524-9 (Print) 978-1-4614-6525-6 (Online). 
[13] A.V. Kostochka, The independent domination number of a cubic 3-connected graph can be much larger than its domination number, Graphs Combin. 9(3) (1993), 235-237.

[14] P.C.B. Lam, W.C. Shiu, and L. Sun, On independent domination number of regular graphs, Discrete Math. 202 (1999), 135-144.

[15] J. Lyle, A note on independent sets in graphs with large minimum degree and small cliques, Electr. J. Comb. 21(2) P2.38 (2014).

[16] J. Lyle, A structural approach for independent domination of regular graphs, Graphs Combin. 31(5) (2015), 1567-1588.

[17] S. O and D.B. West, Cubic graphs with large ratio of independent domination number to domination number, Graphs Combin. 32(2) (2016), 773-776.

[18] N.J. Rad and L. Volkmann, A note on the independent domination number in graphs, Discrete Appl. Math. 161 (2013) 3087-3089.

[19] J. Southey and M.A. Henning, Domination versus independent domination in cubic graphs, Discrete Math. 313(11) (2013), 1212-1220.

[20] S. Wang and B. Wei, A note on the independent domination number versus the domination number in bipartite graphs, Czechoslovak Math. J. 67(2) (2017), 533-536. 